\title{
Präventionschecklisten im OP und in der Endoskopie
}

\author{
Wolfram von Bernstorffa Tilman Pickartz $^{b} \quad$ Alexandra Busemann $^{a, c}$ \\ ${ }^{\text {a} A b t e i l u n g ~ f u ̈ r ~ A l l g e m e i n e ~ C h i r u r g i e, ~ V i s z e r a l-, ~ T h o r a x-~ u n d ~ G e f a ̈ ß c h i r u r g i e, ~ K l i n i k ~ u n d ~ P o l i k l i n i k ~ f u ̈ r ~ C h i r u r g i e, ~}$ \\ ${ }^{b}$ Klinik und Poliklinik für Innere Medizin A, Zentrum für Innere Medizin, Universitätsmedizin Greifswald, \\ ${ }^{\circ}$ Chirurgische Arbeitsgemeinschaft für Qualität und Sicherheit in der Chirurgie (CAOS) der Deutschen Gesellschaft für Chirurgie (DGCh), \\ Deutschland
}

\section{Schlüsselwörter \\ OP-Checkliste $\cdot$ Checkliste Endoskopie . \\ Patientensicherheit · WHO-Checkliste}

\section{Zusammenfassung}

Hintergrund: Unerwünschte Ereignisse oder sogar folgenschwere Fehlleistungen entstehen in der Medizin nicht zufällig. Analog zu anderen hochriskanten Arbeitswelten wie der Luftfahrt oder der Kernenergie stellen sie eine Verkettung unglücklicher Umstände bzw. den Zusammenbruch der Multibarrierenstrategie dar. Aus diesem Grund wurden im Laufe der letzten Jahre Checklisten etabliert, die als Teil dieser Sicherheitsbarrieren die Patientensicherheit erhöhen. Methode: Zur Literaturrecherche wurden die Datenbanken von Medline und PubMed sowie der Internetsuchdienst von Google unter den Suchworten "Checkliste», "Sicherheitscheckliste», zum Teil kombiniert mit "Endoskopie» bzw. "Endoscopy", "Surgical Safety Checklist», "Safety Checklist», "Checklist", "Safety Management" und "WHO-Checklist(e)» abgefragt. Die so identifizierten Publikationen wurden im Folgenden auf die relevanten endoskopischen und chirurgischen Belange hin zusammengestellt und um eigene Erfahrungen aus der Chirurgie und Endoskopie ergänzt. Ergebnisse: Operationschecklisten sind nachweislich effektive Instrumente zur Erhöhung der Patientensicherheit und Vermeidung von unerwünschten Ereignissen. Dies ist insbesondere auf eine verbesserte Kommunikation bzw. Teaminteraktion zurückzuführen, mit dem Effekt einer dadurch veränderten Sicherheitskultur. Voraussetzung ist ein gutes Checklistendesign und die gelebte Anwendung der Checklisten durch die Leitungsebenen. Schlussfolgerungen: Operationssaal(OP)-Checklisten verbessern nachhaltig die Sicherheitskultur und erhöhen dadurch die Patientensicherheit. Diese positiven Ergebnisse sollten zunehmend in andere Bereiche der Medizin wie die Endoskopie transferiert werden.

\section{Keywords \\ Surgical safety checklist - Checklist endoscopy . Patient safety - WHO checklist \\ Summary \\ Prevention Checklists in Surgery and Endoscopy}

Background: Adverse or even fatal events in medicine do not happen by chance. They usually result from a concatenation of circumstances, also called the failure of the multi-barrier strategy. This has also been shown for other high-risk working environments like aviation or nuclear power plants. In the past few years, checklists have been developed to increase patient safety. These checklists are part of the patient safety barriers. Method: In the search for relevant publications, the online services of Medline and PubMed as well as Google were used. The keywords used comprised 'Checkliste', 'Sicherheitscheckliste', also combined with 'Endoskopie' or 'Endoscopy', 'Surgical Safety Checklist', 'Safety Checklist', 'Checklist', 'Safety Management' and/or 'WHO-Checklist(e)'. Relevant publications concerning endoscopy and surgery were selected and supplemented by our own experiences in surgery and endoscopy. Results: Surgical checklists have been shown to be effective instruments in increasing patient safety as well as in avoiding undesired events. These effects are due to improved communication and interaction between the team members involved, leading to a significantly improved safety culture. Requirements for successful checklists are their optimal design as well as their conscientious and continuous implementation and application, including the responsible involvement of all seniors and the upper management. Conclusions: Surgical checklists provide lasting improvements of the safety culture and patient safety. Their positive effects should be transferred to other medical fields including specialties like endoscopy.

\begin{tabular}{ll}
\hline KARGER & ( ) 2013 S. Karger GmbH, Freiburg \\
1662-6664/13/0293-0143\$38.00/0 \\
$\begin{array}{l}\text { Fax +49 761 452 07 14 } \\
\text { Information@Karger.com } \\
\text { www.karger.com }\end{array}$ & $\begin{array}{l}\text { Accessible online at: } \\
\text { www.karger.com/vim }\end{array}$
\end{tabular}




\section{Einleitung}

Hochriskante Arbeitswelten mit komplexen Tätigkeiten und Abläufen, an denen viele Personen und Systeme beteiligt sind, erfordern ein reibungsloses Zusammenspiel aller Beteiligten. Dabei werden Fehlleistungen oder Versäumnisse von Einzelnen in der Regel durch andere Beteiligte bzw. automatisierte Kontrollinstanzen kompensiert. Aus einer Gefahr resultiert nur dann ein unerwünschtes Ereignis, wenn die Sicherheitssysteme Schwachstellen aufweisen und diese, wie bei hintereinandergeschalteten Löchern eines Schweizer Käses, in einer Reihe liegen (Abb. 1) [1]. Um die Wahrscheinlichkeit solcher verketteter Fehler weitestgehend zu minimieren, wurden in vielen Bereichen, in denen daraus fatale Konsequenzen für Leib und Leben bzw. die Umwelt resultieren können, entsprechende Kontrollmechanismen eingeführt. So sind in den Risikobereichen von Atomkraftwerken mit sogenannten «Checklisten» entsprechende Sicherheitsbarrieren eingeführt worden, die die Handlungsabläufe im Routinefall, vor allem aber bei Störfällen, verbindlich festschreiben. Gleichermaßen müssen Piloten im Luftverkehr vor Beginn eines jeden Fluges mit «Checklisten» die Sicherheit und Funktionsfähigkeit der entsprechenden Systeme des Flugzeugs überprüfen und feststellen [2,3]. Auch im Falle einer Ausnahmesituation wie dem Ausfall beider Triebwerke strukturieren Checklisten das Notfallmanagement ohne Zeitverlust. Fehlen in den genannten Bereichen diese Kontrollmechanismen, werden sie nur unzureichend durchgeführt oder versagen, dann besteht die Gefahr katastrophaler Auswirkungen. In der Regel resultieren fatale Fehlleistungen in komplexen Arbeitswelten im Sinne aktiver Fehler aus Problemen der Teamarbeit, aus unzureichender oder fehlerhafter Kommunikation, Selbstüberschätzung oder Zuwiderhandlungen. Ebenso können Probleme im Management oder Organisationsablauf im Sinne latenter Fehler die folgenschweren Auswirkungen nach sich ziehen [4, 5].

Die heute gebräuchlichen Operationssaal(OP)-Checklisten wurden in Anlehnung an die Checklisten aus der Luftfahrt erstellt. Eines der Kernelemente ist das Teambriefing (Team Time-out), bei dem sich die beteiligten Personen nochmals mit Namen und Funktion einander bekannt machen und die wichtigen Informationen austauschen und überprüfen. Der daraus resultierende Haupteffekt, nämlich Teambildung durch Identifikation in und mit der Arbeitsgruppe, verbessert in kritischen Situationen entscheidend die Teamleistung durch enge Kooperation der «Mannschaftsmitglieder» und Motivation des Einzelnen, wodurch schließlich die Qualität der Arbeit und des endgültigen Ergebnisses entscheidend verbessert wird.

\section{Vorgehensweise}

Die Online-Datenbanken von Medline und PubMed sowie der Internetsuchdienst von Google wurden unter den Such-

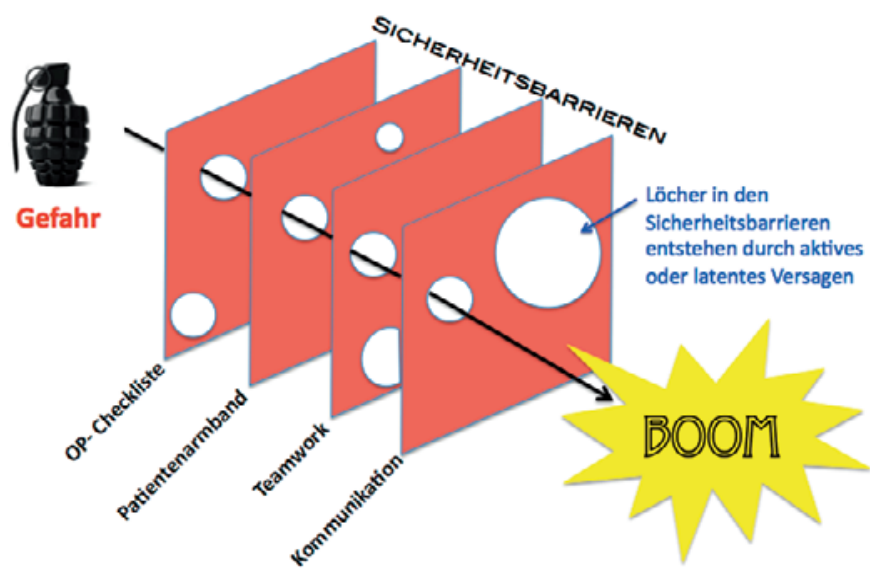

Abb. 1. Schweizer-Käse-Modell der Fehlertheorie nach Reason [1]: Arbeitsabläufe in hochriskanten Bereichen sind im Regelfall durch verschiedene Sicherheitsbarrieren vor Fehlern geschützt. Nur wenn die Sicherheitsbarrieren Lücken aufweisen (z.B. eine unvollständig ausgefüllte OP-Checkliste) und diese Lücken in Reihe geschaltet sind, kann aus einer Gefahr ein unerwünschtes Ereignis resultieren.

worten «Checkliste», «Sicherheitscheckliste», zum Teil kombiniert mit «Endoskopie» bzw. «Endoscopy», außerdem «Surgical Safety Checklist», «Safety Checklist», «Checklist», «Safety Management» und «WHO-Checklist(e)» abgefragt; anschlieBend wurde eine Auswahl der erhaltenen Literatur getroffen. Diese Artikel wurden im Folgenden auf die endoskopischen und chirurgischen Belange hin zusammengestellt und um eigene Erfahrungen aus der Chirurgie und Endoskopie ergänzt.

\section{Ergebnisse}

Im Gesundheitswesen finden komplexe Prozessabläufe mit weitreichenden Folgen für den Einzelnen statt. Aufgrund der enormen Anzahl täglich weltweit stattfindender Operationen mit den entsprechenden Komplikationsmöglichkeiten hat die World Health Organization (WHO) diese Problematik erstmals in einem Pilotprojekt gemeinsam mit einer amerikanischen Universität adressiert. Die Einführung der «Chirurgischen Sicherheits-Checkliste» («Surgical Safety Checklist») wurde an insgesamt acht Krankenhäusern in den westlichen Industrieländern und den Entwicklungsländern erprobt. Mit diesem wenig aufwendigen Mittel konnte bereits eine statistisch messbare Verminderung der operativen Komplikationen von 11 auf 7\% erreicht werden. Ebenso ging die Gesamtsterblichkeit von 1,5 auf 0,8\% zurück. Kern der Checkliste sind einzelne Überprüfungsschritte vor Anästhesiebeginn ( «Signin»), vor der ersten operativen Maßnahme, dem «Schnitt» («Time-out»), sowie nach Beendigung der Operation, der «Naht» («Sign-out»). An jedem einzelnen der drei kritischen Punkte wird der Arbeitsprozess unterbrochen und das Vorgehen bzw. die Unterpunkte mit den Teammitgliedern besprochen und entsprechend abgehakt. Erst nach Abzeichnung des jeweiligen Punktes durch eines der Teammitglieder kann der 


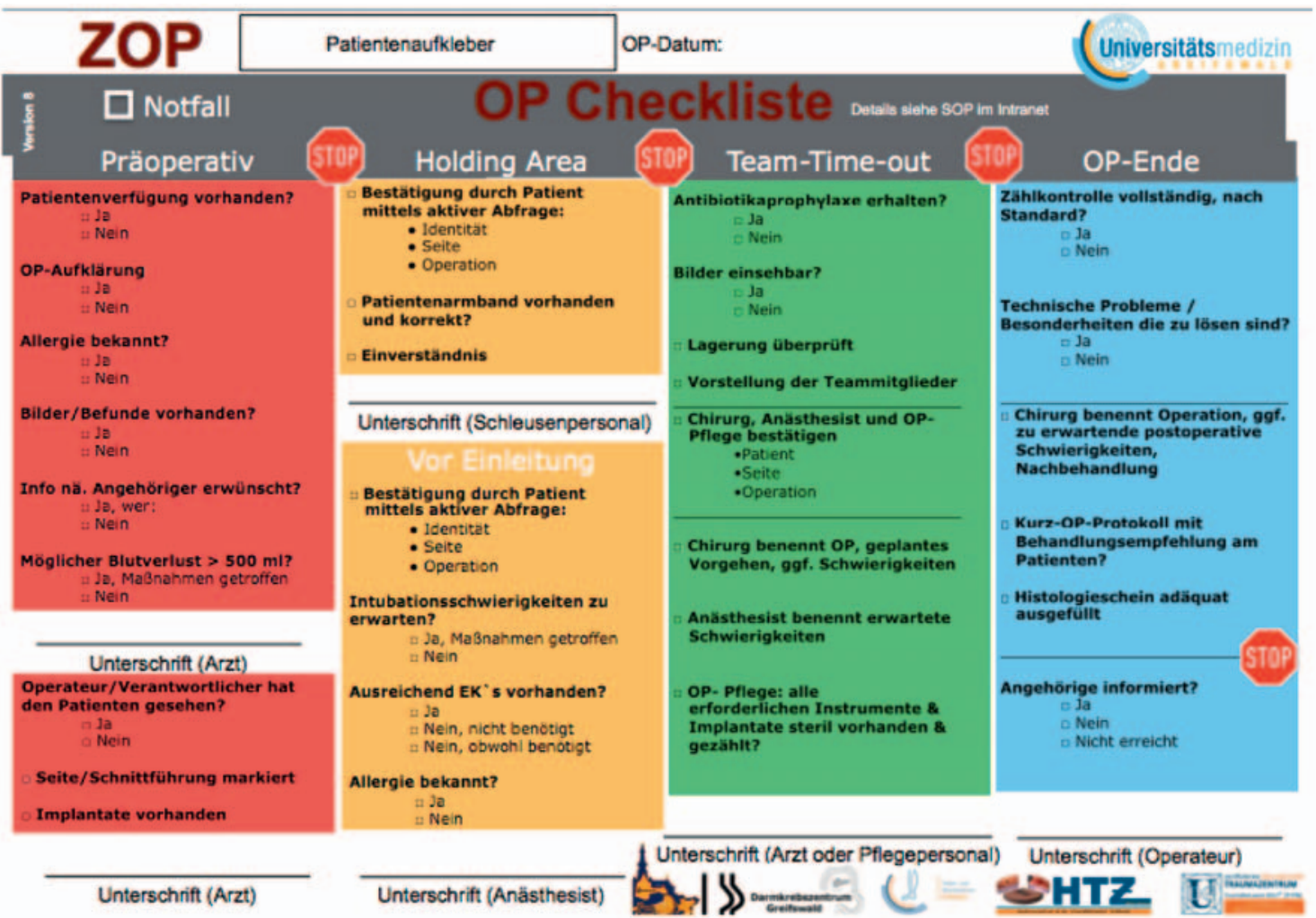

Arbeitsprozess weitergeführt werden [6]. Aufgrund der positiven Ergebnisse hat die WHO die Empfehlung ausgesprochen, diese Checkliste für alle operativen Eingriffe anzuwenden und gleichermaßen alle auszubildenden Fachkräfte von Anbeginn hierin zu schulen [7]. Allerdings sollte diese Liste den Anforderungen der jeweiligen Klinik und Situation angepasst werden [8].

Die Veränderungen in unserem Gesellschaftssystem haben zu einem wachsenden Selbstbewusstsein geführt. Das Internet stellt den Patienten viele, wenn auch nicht immer nützliche, manchmal auch medizinisch wenig sinnvolle oder gar unaufrichtige Informationen zur Verfügung. Gleichzeitig findet eine fortschreitende Stärkung der Patienten gegenüber der Ärzteschaft statt. Zusätzlich finden Meldungen der Medien über «Kunstfehler», «Profitgier» und «verpfuschte Patienten» bei medizinisch oft Halbgebildeten schnell ihren Weg in das allgemeine Bewusstsein. Daher haben der Umgang mit Fehlern, die «Patientensicherheit», das Sicherheitsbewusstsein sowie die generelle Fehler- und Sicherheitskultur zunehmend an Bedeutung gewonnen. Mittlerweile haben sich auch interdisziplinäre und interprofessionelle Gremien unter Einbeziehung der Patienten zu diesem Thema fest etabliert [9]. Die chirurgischen Disziplinen sind mittlerweile zu Vorreitern in der Erprobung und im Einsatz von Checklisten geworden. In Deutschland hat sich nach Veröffentlichung der WHOSicherheitscheckliste im Jahr 2009 deren Gebrauch in Originalform oder in modifizierter Form weitgehend etabliert.

Allerdings konnte auch in nichtchirurgischen medizinischen Bereichen gezeigt werden, dass die Einführung von Si- cherheitschecklisten für gezielte Problemfelder die Patientensicherheit erhöht. So berichtete eine englische Arbeitsgruppe vom erfolgreichen Einsatz einer Checkliste, die das Thrombembolierisiko eines Patienten erfasst. Infolge des Einsatzes dieser Checkliste kam es zu signifikant weniger thrombembolischen Ereignissen [10]. In der Pflege sind stichpunktartig konfigurierte Überleitungsbögen in Checklistenform seit langem etabliert. Eine US-amerikanische Gruppe konnte zeigen, dass der Einsatz entsprechend modifizierter Überleitungsprotokolle für den ärztlichen Bereich signifikant die Qualität des Informationsflusses bei der Patientenübergabe verbessert und zur Sicherheit in der Patientenversorgung beiträgt [11].

Auch in endoskopischen Abteilungen finden Sicherheitschecklisten immer mehr Verbreitung. In der gastrointestinalen Endoskopie werden die diagnostischen Untersuchungen im Verlauf der vergangenen Jahre durch immer mehr komplexe therapeutische Eingriffe ergänzt. Diese weisen teilweise eine erhebliche Invasivität auf, wie z.B. die endoskopische Submukosaresektion (ESD), die endoskopische transmurale Pankreasnekrosektomie oder neuere Verfahren wie POEM (peroral endoscopic myotomy) oder SET (submucosal endoscopic tumor resection). Die technischen Ansprüche sowohl an das Personal als auch an die verwendeten Materialien sind sehr hoch. Zudem sind diese Eingriffe teilweise zeitintensiv, was sie auch in Bezug auf die Sedierung sehr anspruchsvoll werden lässt. Vor diesem Hintergrund ist der Einsatz von Sicherheitschecklisten in der Endoskopie nur eine logische Konsequenz.

Die gastrointestinale Endoskopie zeichnet sich unter anderem durch eine Reihe von redundanten Spezifika aus, die 

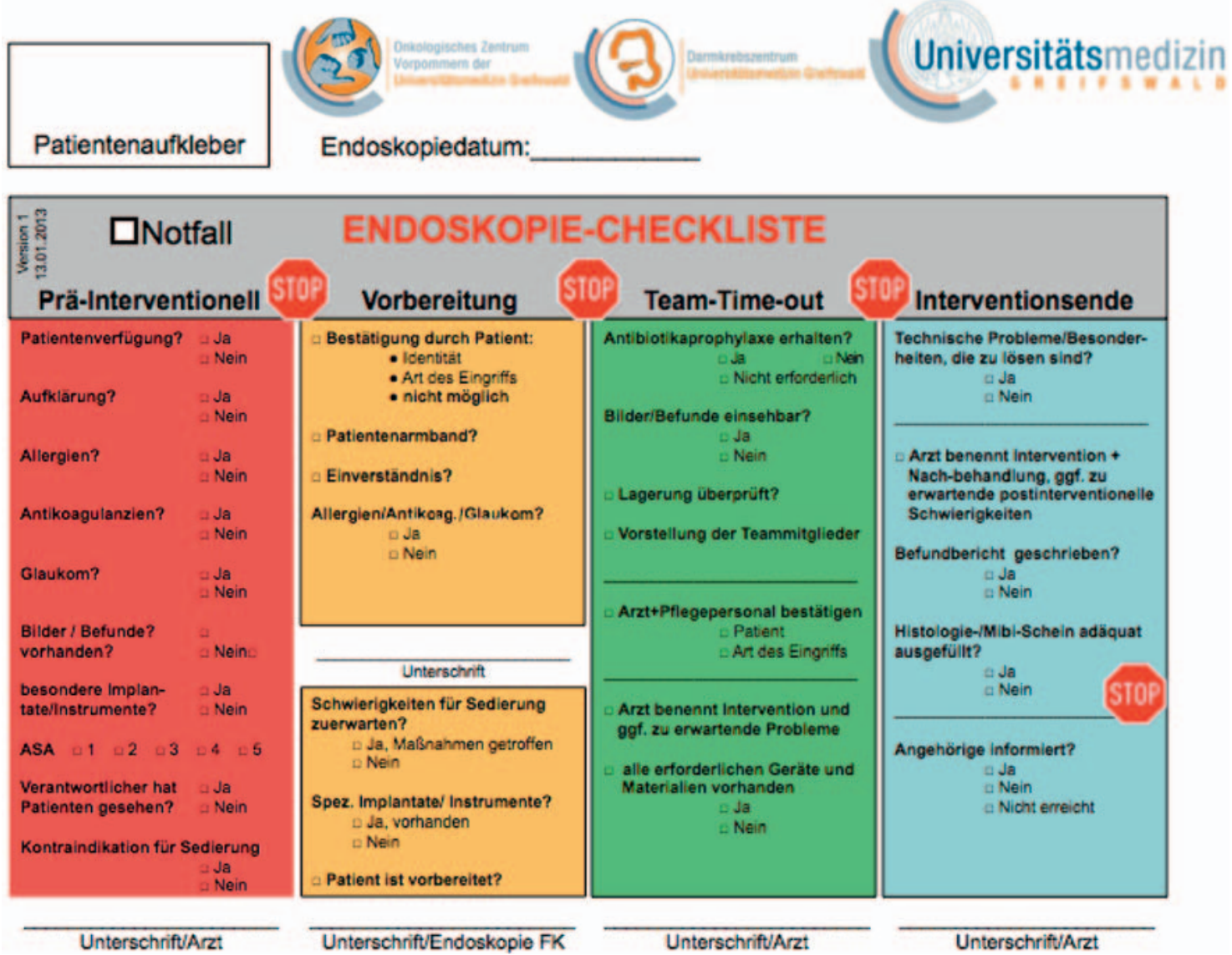

Abb. 3. EndoskopieCheckliste in Anlehnung an die Greifswalder WHO-OP-

Checkliste. somit in die Checklisten fest implementiert werden müssen. So werden regelmäßig Spasmolytika benötigt, deren Einsatz den zumindest anamnestischen Ausschluss eines Glaukoms erfordert. Entgegen einer chirurgischen Operation werden die wenigsten Eingriffe unter sterilen Bedingungen durchgeführt, was die Unschärfe zwischen untersuchendem und assistierendem Personal, insbesondere in Notfallsituationen, erhöht und somit eine klare «Rollenverteilung» erfordert. Darüber hinaus findet in den wenigsten Fällen eine zusätzliche fachärztliche anästhesiologische Begleitung statt und nur sehr wenige Eingriffe erfolgen unter Intubation und Beatmung. Nach entsprechender Schulung übernimmt pflegerisches Assistenzpersonal in einigen Institutionen auch die Verabreichung von Sedativa [12]. Hier ist eine sorgfältige Einschätzung des Sedierungsrisikos erforderlich und die erforderlichen Maßnahmen sind zu ergreifen. Inhaltlich trägt diesem letzten Punkt z.B. die S3Leitlinie «Sedierung in der gastrointestinalen Endoskopie» Rechnung [13]. Grundsätzlich ist die durchschnittliche Dauer einer endoskopischen Untersuchung bzw. eines endoskopischen Eingriffs kürzer und der personelle Aufwand geringer als bei einem chirurgischen Eingriff. Dieser Umstand sollte in der Ausgestaltung einer Checkliste ebenfalls berücksichtigt werden, um den Aufwand auf ein Maß zu reduzieren, das eine maximale Akzeptanz ermöglicht.

Harte Daten zur Senkung der Morbidität und Mortalität durch die Einführung von Sicherheitschecklisten in der gast- rointestinalen Endoskopie liegen zum gegenwärtigen Zeitpunkt noch nicht vor. Die American Society of Gastrointestinal Endoscopy schlägt auf ihrer Homepage eine Version einer Sicherheitscheckliste für die Endoskopie vor, die in Anlehnung an die WHO-Checkliste für die Chirurgie erarbeitet wurde [14]. Ebenfalls in Anlehnung an diese veröffentlichen einige einzelne endoskopierende Abteilungen adaptierte Checklisten [15].

In unserem Universitätsklinikum wird seit Gründung einer interdisziplinären Abteilung für gastrointestinale Endoskopie unter Mitarbeit von Gastroenterologen und Chirurgen ein Protokoll verwendet, das die wesentlichen und relevanten Sicherheitsaspekte in relativ kurzer Form berücksichtigt und das vor jeder Untersuchung zur Anwendung kommt. Dieses Protokoll wird sowohl vom Untersucher als auch vom Assistenzpersonal überprüft und unterschrieben. Unsere eigene Erfahrung zeigt seither einen deutlich bewussteren Umgang mit den für die Endoskopie sicherheitsrelevanten Fragen. In Anlehnung an die bereits seit längerem etablierte und verwendete Checkliste in der Chirurgie (Abb. 2) haben wir eine endoskopische Checkliste erarbeitet, die in Abbildung 3 dargestellt ist. Diese könnte in Zukunft alternativ oder ergänzend Verwendung finden.

In der Universitätsmedizin Greifswald konnte in der Klinik für Chirurgie Anfang 2009 eine angepasste Form der WHOOP-Checkliste (Abb. 2) eingeführt werden. Auslöser hierfür 


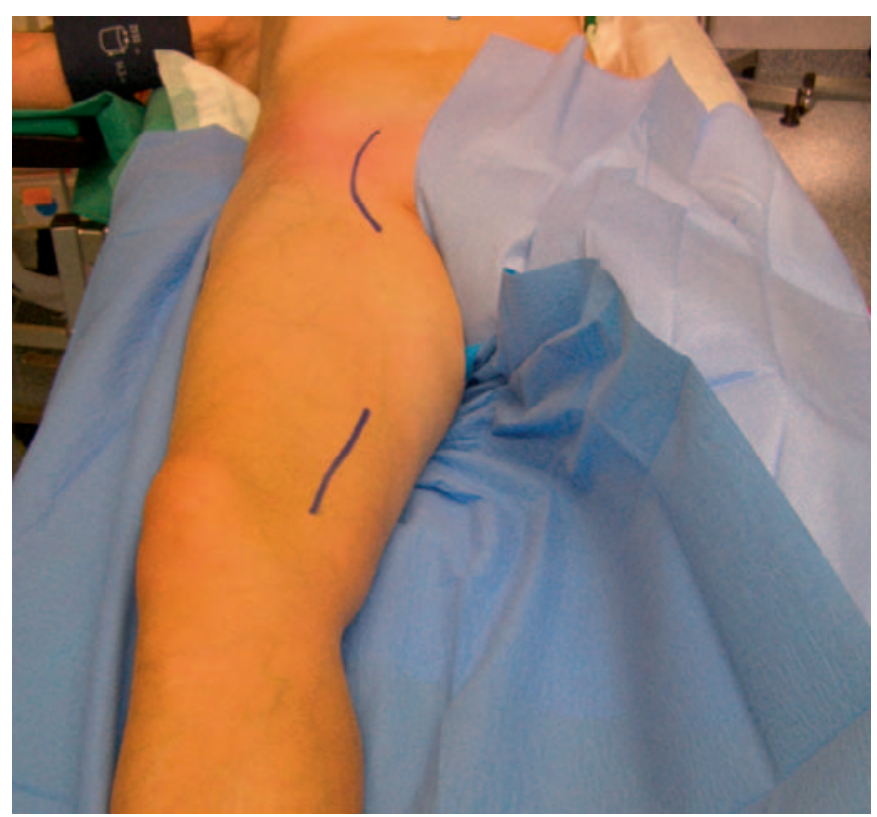

Abb. 4. Anzeichnen der Schnittführung zur Anlage eines P1-Gefäßbypasses: Die Seite und die Art der Operation sind eindeutig markiert und das Darlegen der genauen Schnittführung gibt dem Patienten die Möglichkeit zur Nachfrage.

war der in London durchgeführte Workshop «Safe Surgery Saves Lives». Verschiedene kritische Punkte waren für unsere Klinik hierbei zwingende Voraussetzung. Zunächst muss der Patient von «seinem» Operateur persönlich vor der Operation gesehen werden. Dieser hat die Indikation für die Operation $\mathrm{zu}$ bestätigen und sollte dies noch einmal kurz mit dem Patienten erläutern. Weiterhin war auf der Liste die durchgeführte Aufklärung anhand eines entsprechenden Protokolls zu bestätigen. Da diese Punkte nicht direkt vor Anästhesiebeginn geprüft werden können - ein Verneinen dieser Punkte würde zum kompletten Abbrechen des Prozesses führen -, musste hier eine weitere Säule zu den ursprünglichen drei Säulen (Sign-in - Time-out - Sign-out) eingeführt werden. Diese ist am Tage vor der Operation bzw. noch in der Ambulanz zu bearbeiten. Ein wesentlicher Punkt für Patienten und Chirurgen ist hierbei das Anzeichnen der Operationsseite des Patienten. Im Laufe der Evaluation der Checkliste zeigte sich, dass die Markierung der tatsächlichen Schnittführung sowie möglicher Erweiterungen wesentlich zum Verständnis des Patienten beiträgt, da er das Ausmaß der Operation unmittelbarer begreift. Somit kann zusätzliches Vertrauen geschaffen werden, die Unverwechselbarkeit des Patienten im Sinne der Patientensicherheit nimmt zu und die Sicherheit für den Eingriff steigt erheblich. Außerdem kann der Patient aufkommende Fragen unverzüglich mit dem Chirurgen klären, ohne dass der eigentliche Prozessablauf am Operationstag darunter leidet (Abb. 4).

Unsere chirurgische Checkliste in der Greifswalder Form ist mittlerweile in der 8. Version in täglicher Verwendung und zeugt damit von der kontinuierlichen Überprüfung und Ver- besserung unseres Ablaufes. Anfangs bestand eine erhebliche Skepsis gegenüber der Liste, die sich auch in vielen Publikationen widerspiegelt. Die Angst der Chirurgen vor einer Kontrolle und Bevormundung durch die Operationsschwester spielt ebenso eine Rolle wie der mögliche Anspruch des Chirurgen auf Fehlerlosigkeit und der aus diesen Punkten folgenden eventuell gefühlten Minderwertigkeit und Kränkung [16]. Zusätzlich erscheint vielen Beteiligten die mehrfache Kontrolle überflüssig, zeitraubend und lästig und die namentliche Vorstellung im Operationssaal wird als «fremd» oder seltsam empfunden. Eine Checkliste mit entsprechender Überprüfung der einzelnen Punkte erfordert ein hohes Maß an Selbstdisziplin des Operationsteams, vom Anästhesisten über die Anästhesiepfleger und OP-Schwestern bis zum Chirurgen. Die überwiegende Mehrheit der Beteiligten ist davon überzeugt, dass die Checkliste vor chirurgischen Fehlern und Komplikationen schützen kann. Zusätzlich äußerten die Studienteilnehmer den Wunsch, dass bei Operationen der eigenen Person eine ebensolche Checkliste verwendet wird [17]. Von den chirurgischen Kollegen, dem OP-Pflegepersonal sowie den Anästhesisten und den entsprechenden Pflegekräften an unserer Klinik ist sie, nach anfänglichen Zweifeln und Widerständen, voll integriert und akzeptiert und hat zu einer Verbesserung der Abläufe und zur Vermeidung von Fehlern und Komplikationen geführt.

Dies konnte ebenso für dringliche Eingriffe an einem großen Kollektiv von über 1700 Patienten gezeigt werden. Vor Einführung der WHO-Checkliste lag die Komplikationsrate bei $18,5 \%$ und konnte auf $11,7 \%$ nach Inauguration der Checkliste gesenkt werden. Gleichermaßen fiel der Anteil der Sterblichkeit signifikant von 3,7 auf 1,4\% [18]. Eine weitere Studie bestätigte diese günstigen Ergebnisse an einem retrospektiven Kollektiv von über 250000 Patienten [19]. Interessanterweise hatte auch die korrekte Durchführung/Bearbeitung der einzelnen Säulen der Checkliste einen entscheidenden Einfluss auf die Sterblichkeitsraten.

Gleichermaßen wirkt sich die Umsetzung einer Checkliste positiv auf den Umgang mit Fehlern, die «gelebte» Sicherheit sowie auf das Sicherheitsempfinden, also auf die Fehler- und Sicherheitskultur, aus. Dies fördert die Bereitschaft, Probleme gemeinsam zu erkennen und Lösungswege zu erarbeiten. Ebenso steigt die Frequenz der Diskussion kritischer Vorfälle zwischen Chirurgen und Narkoseärzten [20-22]. Allerdings müssen die elementaren Teile der Checkliste auch dauerhaft und gewissenhaft erfüllt werden. Leider zeigt sich immer wieder, dass die Vollständigkeit ihrer Einhaltung häufig im Laufe der Anwendungszeit abnimmt [23, 24]. Ebenso scheint vor allem die dritte Säule («Sign-out») häufiger als die anderen vernachlässigt zu werden [25]. Schließlich zeigte eine Studie, dass trotz auffälliger präoperativer Ereignisse oder Ergebnisse in über $2 \%$ der Fälle dennoch mit der Operation begonnen wurde [26]. Spezielle Trainingseinheiten und Fortbildung der jeweiligen Beteiligten, ähnlich denen von Flugzeugbesatzungen, werden die Verwendung der Checkliste signifikant 
verbessern und viele der angesprochenen Mängel abstellen. Vor allem durch eine vollständige Durchdringung aller Mitarbeiter, insbesondere der höchsten Leitungsebene der Kliniken, sowie die Einführung von Checklistenbeauftragen werden deutliche Verbesserungen bei der Umsetzung der Checkliste erzielt [27-30]. Nach der Einführung von Checklisten darf aber die Wachsamkeit der Benutzer nicht im Laufe der Zeit abnehmen. Unregelmäßigkeiten bei der Anwendung können ein vorgetäuschtes und falsch verstandenes Sicherheitsgefühl hervorrufen, sodass die positiven Auswirkungen der Liste sich leicht in ihr Gegenteil verkehren und damit vermehrt zu Fehlern führen können. Um alle vorgenannten Fehlerquellen zu minimieren und die Vorteile umzusetzen, sollten Checklisten bestimmte Eigenschaften aufweisen. Sie sollten nicht mehr als eine Seite einnehmen und nur Punkte beinhalten, die bei Nichteinhalten eine hohe Gefahr für den Patienten darstellen, sogenannte «common killers». Idealerweise sollte die Bearbeitung nicht mehr als 90 Sekunden beanspruchen. Sind im Einzelfall Kontrollpunkte zu modifizieren oder müssen diese verändert oder verbessert werden, sollte dies möglichst nicht zum Abbruch des Gesamtablaufes der jeweiligen Säule führen. Kritisch ist die zeitliche Abfolge der Punkte zu wählen, um Kontrollpunkte vor Tätigkeiten zu wählen, die nicht mehr umkehrbar sind. So werden der Belegschaft entsprechende Verbesserungen oder Veränderungen an die Hand gegeben. Die Liste muss den Erfordernissen vor Ort und den einzelnen Abteilungen bzw. Tätigkeitserfordernissen angepasst werden. Entscheidend für den Erfolg sind die ständige Überprüfung der Anwendungen sowie die regelmäßige Durchführung an die Bedürfnisse angepasster Modifikationen, insbesondere bei relevanten Veränderungen im Ablauf der Tätigkeiten. Schließlich müssen die günstigen Auswirkungen der Checkliste evaluierbar sein [31].

\section{Schlussfolgerungen}

Die Resultate der verfügbaren Studien sowie unsere eigenen Ergebnisse in der Chirurgie zeigen eindrucksvoll, dass OP-Checklisten effektive Mittel sind, um die Sicherheit von Patienten im operativen Alltag signifikant zu erhöhen. Dies führt zu einer Verminderung von Komplikationen, operations- assoziierten Erkrankungen sowie der Sterblichkeit. Gleichzeitig wird die Kooperation zwischen Operationsteam und Anästhesieteam deutlich verbessert, die fachliche Kommunikation erhöht und die kooperative und kollegiale Zusammenarbeit der Mitarbeiter gefördert. Schließlich kommt es zu positiven Effekten auf die Fehler- und Sicherheitskultur in diesen Bereichen. Die Gestaltung der jeweiligen Checkliste ist von besonderer Bedeutung; sie sollte vor allem kurz und leicht verständlich sein. Alle Bereichsmitglieder, die mit der Checkliste arbeiten, müssen von ihrer Sinnhaftigkeit im Alltag überzeugt sein; ein reines «Abhaken» der jeweiligen Punkte muss strikt vermieden werden. Entscheidend für eine erfolgreiche Einführung der Checkliste ist, dass diese von der Leitungsebene gefördert und engagiert selbst angewendet wird. Die Sicherheit kann nur dann gesteigert werden, wenn sie von allen - und vor allem von der Führung - «gelebt» wird.

Sicherheitschecklisten finden auch in endoskopischen Abteilungen immer mehr Beachtung. Die flächendeckende Nutzung einer Sicherheitscheckliste in der Endoskopie ist die logische Konsequenz aus den Erfahrungen und den publizierten Ergebnissen der Nutzung chirurgischer Sicherheitschecklisten. Abbildung 3 zeigt beispielhaft eine solche Checkliste. Entsprechend den Anforderungen eines Qualitätsmanagementsystems muss eine Validierung der konsequent angewandten Checkliste den Status quo ermitteln. Dieses sollte analog der chirurgischen WHO-Checkliste im Rahmen von Studien untersucht und publiziert werden. Diese stehen zum gegenwärtigen Zeitpunkt aus. Im Rahmen des qualitätssichernden Plan-Do-Check-Act(PDCA)-Prozesses muss in der Folge eine optimierte Anpassung an die speziellen Erfordernisse der gastrointestinalen Endoskopie erfolgen. Im Sinne eines Benchmarkings sollten Bewertungskriterien einer flächendeckend möglichst einheitlichen Sicherheitscheckliste erarbeitet werden. Die wesentliche Orientierungsgröße in dem Bestreben, diese Checkliste zu verbessern, muss eine möglichst geringe Komplikationsrate sein.

\section{Disclosure Statement}

Die korrespondierende Autorin gibt für sich und ihre Koautoren an, dass kein Interessenkonflikt besteht.

\section{Literatur}

1 Reason J: Human error: models and management. BMJ 2000;320:768-770.

2 Turner JW, Huntley MS: The Use and Design of Flight Crew Checklists and Manuals (DOT/FAA/ AM-91/7). Cambridge, National Transportation System Center, 1991.

3 Degani A, Wiener EL: Cockpit checklists: concepts, design and use. Hum Factors 1993;35:345-359.

4 Dekker S: Field Guide to Understanding Human Error. Hampshire, Ashgate Publishing, 2006.
5 Kohn LT, Corrigan JM, Donaldson MS: To Err is Human: Building a Safer Health System. Washington, National Academy Press, 2000.

6 Haynes AB, Weiser TG, Berry WR, et al: A surgical safety checklist to reduce morbidity and mortality in a global population. N Engl J Med 2009;360: 491-499.

7 www.who.int/patientsafety/safesurgery/en/.

8 www.who.int/patientsafety/safesurgery/ss_checklist/ en/index.html.
9 www.aktionsbuendnis-patientensicherheit.de.

10 Colborne NR, Lake DR, Wear KR, Thomson GA: Using a venous thromboembolism checklist significantly improves VTE prevention: a junior doctor led intervention. Int J Clin Pract 2013;67:157-160.

11 Abraham J, Kannampallil T, Patel B, Almoosa K, Patel VL: Ensuring patient safety in care transitions: an empirical evaluation of a handoff intervention tool. AMIA Annu Symp Proc 2012;2012: $17-26$. 
12 Dumonceau JM, Riphaus A, Aparicio JR, et al European Society of Gastrointestinal Endoscopy, European Society of Gastroenterology and Endoscopy Nurses and Associates, and the European Society of Anaesthesiology Guideline: Non-anesthesiologist administration of propofol for GI endoscopy. Endoscopy 2010;42:960-974.

13 Riphaus A, Wehrmann T, Weber B, et al: S3-Leitlinie - «Sedierung in der gastrointestinalen Endoskopie». Z Gastroenterol 2008;46:1298-1330.

14 www.asge.org/WorkArea/showcontent.aspx?id= 14262.

15 www.safesurg.org/uploads/1/0/9/0/1090835/kirkland __endoscopy_2.pdf.

16 Cabarrot P, Bataillon R, Le Moign R: One year implementation of the safe surgery checklist in France, what has been achieved so far, what could be improved? (article in French). Ann Fr Anesth Reanim 2011:30:469-474.

17 Allard J, Bleakley A, Hobbs A, Coombes L: Presurgery briefings and safety climate in the operating theatre. BMJ Qual Saf 2011;20:711-717.

18 Weiser TG, Haynes AB, Dziekan G, Berry WR Lipsitz SR, Gawande AA: Effect of a 19-item surgical safety checklist during urgent operations in a global patient population. Ann Surg 2010;251:976980.
19 van Klei WA, Hoff RG, van Aarnhem EE, et al: Effects of the introduction of the WHO «Surgical Safety Checklist» on in-hospital mortality: a cohort study. Ann Surg 2012;255:44-49.

20 Haynes AB, Weiser TG, Berry WR, et al: Changes in safety attitude and relationship to decreased postoperative morbidity and mortality following implementation of a checklist-based surgical safety intervention. BMJ Qual Saf 2011;20:102-107.

21 Nilsson L, Lindberget O, Gupta A, Vegfors M: Implementing a pre-operative checklist to increase patient safety: a 1-year follow-up of personnel attitudes. Acta Anaesthesiol Scand 2010;54:176-182.

22 Helmio P, Blomgren K, Takala A, Pauniaho SL, Takala RS, Ikonen TS: Towards better patient safety: WHO surgical safety checklist in otorhinolaryngology. Clin Otolaryngol 2011;36:242-247.

23 Paugam-Burtz C, Guerrero O: French surgical checklist in a university hospital: achievements one year after implementation (article in French). Ann Fr Anesth Reanim 2011;30:475-478.

24 Vogts N, Hannam JA, Merry AF, Mitchell SJ: Compliance and quality in administration of a surgical safety checklist in a tertiary New Zealand hospital. N Z Med J 2011;124:48-58.
25 Gueguen T, Coevoet V, Mougeot M, et al: Deployment of the checklist «Patient safety in the operating room» in two Lorraine hospitals. Performances and difficulties (article in French). Ann Fr Anesth Reanim 2011;30:489-494.

26 Rateau F, Levraut L, Colombel AL, et al: Checklist «Patient Safety» in the operating room: one year experience of 40,000 surgical procedures at the university hospital of Nice (article in French). Ann Fr Anesth Reanim 2011;30:479-483.

27 Conley DM, Singer SJ, Edmondson L, Berry WR, Gawande AA: Effective surgical safety checklist implementation. J Am Coll Surg 2011;212:873-879.

28 Mahajan RP: The WHO surgical checklist. Best Pract Res Clin Anaesthesiol 2011;25:161-168.

29 Paull DE, Mazzia LM, Izu BS, Neily J, Mills PD, Bagian JP: Predictors of successful implementation of preoperative briefings and postoperative debriefings after medical team training. Am J Surg 2009;198:675-678.

30 Bosk CL, Dixon-Woods M, Goeschel CA, Pronovost PJ: Reality check for checklists. Lancet 2009 374:444-445.

31 Weiser TG, Haynes AB, Lashoher A, et al: Perspectives in quality: designing the WHO surgical safety checklist. Int J Qual Health Care 2010;22: 365-370. 